\title{
ANALYSIS OF SWEPT PLATES WITH STRUCTURAL REDUCTION USING TRANSITION ELEMENT CONCEPT
}

\author{
B. R. SOMASHEKaR $\dagger$ \\ National Aeronautical Laboratory. Bangalore 560017. India
}

and

C. S. NANJUNDA RaM $\ddagger$

Malnad College of Engineering, Hassan 573201, India

(Received 12 January 1982; received for publication 7 March 1983)

\begin{abstract}
Near the fixed end boundaries of cantilever swept plates, a high stress variation, especially in a near singular fashion at the re-entrant corner, requires that at least at this region, one requires an analysis with a high precision triangular plate bending element. An idealisation of an entire swept plate, especially one of large aspect ratio, by high precision elements may be uneconomical and the approximation of regions away from the boundary by a suitable beam element will achieve a considerable reduction in problem size. Thus one can achieve a structural reduction of the swept plate problem by the use of plate elements in regions of high stress variation, beam elements in regions away from the kinematically constrained boundaries and a variationally condensed transition plate/beam element which will have the nodes and degrees of freedom of high precision $C_{1}$ continuous plate elements along one boundary and the node and degrees of freedom of a beam element along the other boundary. This interphase element will provide the required transition from thin plate to thin beam behaviour. In this paper, extensive results indicate a considerable reduction in problem size and solution time without significant loss of accuracy in stresses, displacements and slopes.
\end{abstract}

\section{INTRODUCTION}

A thin plate is a commonly used structural element for many engineering applications. It is essentially a flat structural element with its thickness dimension much smaller than its other 2-dimensions and it resists loads normal to its plane by a purely flexural action. The flexural behaviour of thin plates has received wide analytical interest for over four centuries and it is universally accepted now that the governing equation for a thin isotropic plate is the fourth order bi-harmonic partial differential equation.

In this paper, we are interested in an economical numerical analysis of a cantilever swept plate. Near the fixed end boundary of a cantilever swept plate or in regions of rapid variation of load distribution (e.g. a single concentrated load), the stressing phenomenon is really 3-dimensional. Even if these are approximated by 2-dimensional plate theory, there are high bending stress variations, especially in a near singular fashion at reentrant or abrupt corners, which requires a more accurate analyses in these regions. Again, there are regions of the plate where the stress variations may be smooth to the point of being one dimensional in behaviour and the application of the standard bi-harmonic plate equation in this region may be superfluous. In the major part of a cantilever plate, at sufficient distances away from the fixed end, the plate behaves essentially as a beam and it is adequate if beam theory can be used in this region.

While these types of approaches were mentioned in the literature decades ago, it has not been easy to realise efficient theoretical models within the continuum theories because of the necessity of dealing with different domains with different field equations and matching the kinematic field variables at the interdomain boundaries.

†Head, Structures Division.

$\ddagger$ Professor, Department of Mechanical Engineering.
The finite element method has proved to be the most general and yet the most powerful method of numerical analysis for various structural field problems. Since the basis of the method is the discretisation of field domains by sub-division into very small but non-infinitesimal subdomains, it becomes possible now to model different regions of the structure by different types of elements, each designed to be efficient in solving a particular type of field equation. This paper attempts one such model which combines plate theory and beam theory with provision to use each theory where required and with interdomain transition achieved by a specially designed interphase or transition element.

In this paper, the plate region is modelled by the most commonly used high precision $C_{1}$ continuous triangular plate element of Cowper et al. [1] which has six degrees of freedom at each of the three nodes of the triangle, namely the transverse displacement, its two cartesian first order derivatives and its three cartesian second order derivatives: $W W_{, x} W_{, y} W_{, x x} W_{, x y} W_{, y y}$. The beam region is modelled using a torsion-flexure coupled beam element [2] which will have as degrees of freedom: $W W$, $W_{, y} W_{, x x} W_{, x y}$ in the global assembly and whose local degrees of freedom are: $W \theta_{x} \theta_{x x} \psi_{y} \psi_{y x}$ where $W$ is the transverse displacement, $\theta_{x}$ the rotation of the normal and $\psi_{y}$ the angle of twist.

The next section of this paper will examine the development of the transition element which will be constructed by initially treating the transition region as an assembly of plate elements and by a suitable variationally and kinematically consistent condensation procedure, produce the required nodes and nodal variables to effect the transition form the plate element to the beam element.

\section{TRANSITION ELEMENT}

The transition element is essentially an extension of the idealisation of the plate region ( $I$ of Fig. 1) into the transition region (II of Fig. 1) by the same plate elements. 


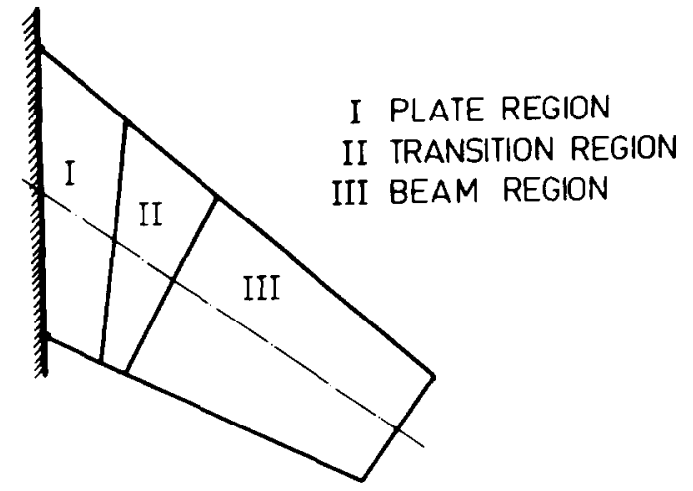

Fig. 1. Division of swept plate into three regions.

As the number of nodes on the plate side of the transition element will be determined by the idealisation to be chosen for the plate region, some preliminary work with the idealisation of the whole plate with the triangular plate elements was necessary [3]. It was determined that five nodes along the plate edge of the transition element offered an acceptable degree of accuracy. Fig. 2(a) shows the idealisation adopted for the transition region. After condensation, the configuration would be as shown in Fig. 2(b). This condensation is achieved by the appropriate introduction of suitable kinematically consistent constraints along the right edge of the transition element so that the thirty degrees of freedom originally available on this edge are constrained to the five at the condensed node by a simple reconstruction using a Taylor series expansion about this node.

Let $\{q\}$ be the vector of global displacements in the transition super-element in Fig. 2(a), where

$$
\{q\}^{T}=\left\{q_{1}{ }^{T}, q_{2}{ }^{T}\right\}
$$

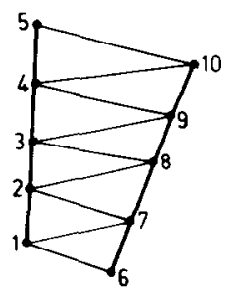

\section{DEGREES OF FREEDOM AT EACH NODE: $W_{, x} w_{, y} w_{, x x} W_{, x y} w_{, y y}$}

Fig. 2(a). Transition element before condensation.
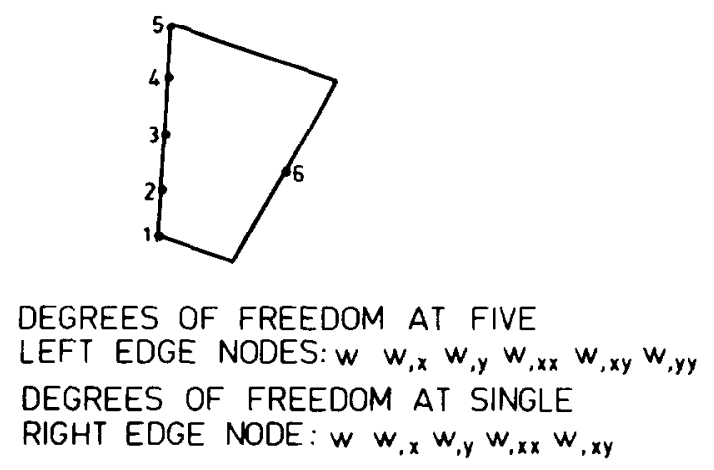

Fig. 2(b). Transition element after condensation. and

$$
\begin{aligned}
\left\{q_{1}\right\}^{T} & =\left\{\delta_{1}{ }^{T}, \delta_{2}{ }^{T}, \delta_{3}{ }^{T}, \delta_{4}{ }^{T}, \delta_{5}{ }^{T}\right\} \\
\left\{q_{2}\right\}^{T} & =\left\{\delta_{6}{ }^{T}, \delta_{7}{ }^{T}, \delta_{8}{ }^{T}, \delta_{9}{ }^{T}, \delta_{10}{ }^{T}\right\}
\end{aligned}
$$

and $\left\{\delta_{j}\right\}$ is the vector of nodal displacements at the $j$ th node defined as

$$
\left\{\delta_{j}{ }^{T}\right\}=\left\{W W_{, x} W_{, y} W_{, x x} W_{x y} W_{, y y}\right\} .
$$

If the assembled stiffness matrix of this region is $[K]$, the total strain energy of this region can be written as

$$
\{U\}=\{q\}^{T}[K]\{q\} .
$$

The vector $\{q\}$ may now be replaced by the vector of global displacements $\{\bar{q}\}$ corresponding to the transition element shown in Fig. 2(a), as

$$
\{\bar{q}\}^{T}=\left\{q_{1}{ }^{T}, \bar{q}_{2}{ }^{T}\right\}
$$

where $\left\{q_{1}\right\}$ remains same as defined in eqn (2) and

$$
\left\{\bar{q}_{2}\right\}^{T}=\left\{\bar{\delta}_{6}^{T}\right\}
$$

and $\left\{\bar{\delta}_{6}{ }^{T}\right\}$ is the vector of nodal displacements at the beam node,

$$
\left\{\bar{\delta}_{6}{ }^{\mathrm{T}}\right\}=\left\{W W, x W_{, y} W,{ }_{x x} W,_{x y}\right\}
$$

If a kinematically consistent transformation from $\left\{q_{2}\right\}$ to $\left\{\bar{q}_{2}\right\}$ is achieved by a transformation matrix [T], then the potential energy of the region may be reconstituted using eqn. (4).

$$
\{U\}=\left\{\bar{q}^{T}\right\}\left[T_{1}\right]^{T}[K]\left[T_{1}\right]\{\bar{q}\}
$$

where

$$
\left[T_{1}\right]=\left[\begin{array}{cc}
{[I]} & 0 \\
0 & {[T]}
\end{array}\right]
$$

is a $60 \times 35$ matrix and $[I]$ is the identity matrix. The details of the derivation of the $[T]$ matrix are given in the Appendix. Clearly, in a variationally consistent way, the stiffness matrix of the transition element after condensation is

$$
[\bar{K}]=\left[T_{1}\right]^{T}[K]\left[T_{1}\right]
$$

In a similar way, the rederived consistent load vector corresponding to the transition element can be obtained as

$$
\{\bar{P}\}=\left[T_{1}\right]^{T}\{P\} .
$$

\section{STRESSES IN SWEPT CANTILEVER PLATES}

The determination of stresses in a swept cantilever plate is of great interest as they find application in many structural situations. The stress analysis of such plates pose considerable difficulties, especially due to the singular stresses that can occur in the obtuse corner where the fixed and free edges meet and the strength of the singularity increases with sweep angle [4]. A review of several analytical methods used in very early investigations is given in [5]. One of the earliest papers to document the 
effect of sweep angle, on tip deflections, rotations and rear spar stresses was that of Long and Bisplinghoff [6]. Early attempts to treat the outboard part of the wing by beam/cantilever approximation were made by Bisch [7] and Hall [8]. Some early analytical work on swept wings was based on Rayleigh-Ritz procedures using an expansion for the deflection function in a product series made up of a linear combination of the normal modes of free-free and clamped-free beams $[9,10]$. However, slow convergence rates for the approximating series, especially at large sweep angles made the method impractical. In another approach, Schuerch [11] and Reissner and Stein [12] assumed the chordwise deflection pattern to be linear and the spanwise variation was determined by a variational approach. Extension to higher order successive approximations for chordwise variations, e.g. parabolic or cubic could be made to obtain better quantitative data on stress.

Recently there has been a revival of interest in this problem, using both analytical and numerical methods. An application of Reissners variational principle [13] was made to demonstrate a faster convergence with greater accuracy in stresses as the principle allows selection of moment functions as well as deflection functions and this permits better satisfaction of free edge conditions. Coull [14] studied the variation of bending and twisting moment at the root of a cantilever plate having a central tip load and it was seen that as the angle of sweep increases, the numerical accuracy also decreases due to the rapid variation of local bending stress. An application of the finite element method was made by Dawe [15]. Parallelogram plate elements are used to model the entire region of the plate and the deflection patterns over several skew angles are presented. However no stresses are presented.

This problem which has a well recorded historical interest serves as an excellent example to demonstrate the usefulness of structural reduction by transition element concept to get accurate stresses and deflections.
4. FINITE ELEMENT MODELLING

The mesh generation scheme adopted for the present study is shown in Fig. 3. Regions $I$ and $I I$ can be controlled by choice of the parameters $S_{P}$ and $S_{T}$ which determine the lengths $0 A, A B$ as fractions of the span $0 C$ and the angles of the lines which determine the left and right edges of the transition element $\alpha$ and $\beta$. Thus in region $I$, the mesh generation scheme allows a graded mesh, so that the rapidly changing behaviour of the stresses at the obtuse junction, especially at higher skew angles can be accommodated. In region $I I I$, an idealisation from one to seven beam elements is permitted.

\section{RESULTS AND DISCUSSIONS}

Table 1 indicates in tabular form, the variation of results obtained for a straight (i.e. unswept) tapered plate of

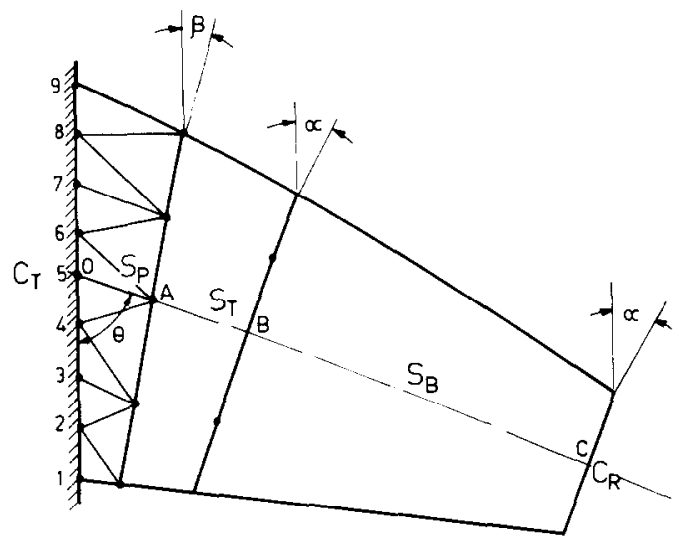

SPAN $=O C$

Fig. 3. Mesh gradation for swept plate.

Table 1. Variation of n.d. tip deflection with aspect ratio for tapered plate $S_{p}=0.05, S_{T}=0.15$

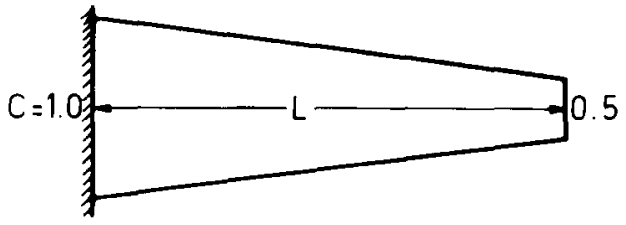

$$
\begin{aligned}
\bar{W} & =\frac{E t^{3} C W}{12 P L^{3}} \text { for } C T L \\
& =\frac{E t^{3} C W}{12 P L^{4}} \text { for udl }
\end{aligned}
$$

\begin{tabular}{|c|c|c|c|c|c|c|c|c|}
\hline \multirow{2}{*}{$L$} & \multicolumn{3}{|c|}{ UNIFORM DISTRIBUTED } & \multicolumn{4}{c|}{ CONCENTRATED } \\
\cline { 2 - 8 } & ALL & \multicolumn{3}{|c|}{ MIXED } & \multicolumn{3}{c|}{ ALL } & \multicolumn{3}{c|}{ MIXED } \\
\hline & COWPER & 7ELE & $5 E L E$ & 3ELE & COWPER & 7ELE & $5 E L E$ & 3ELE \\
\hline 1.5 & 0.0855 & 0.0850 & 0.0855 & 0.0876 & 0.378 & 0.374 & 0.375 & 0.379 \\
\hline 2.0 & 0.0856 & 0.0850 & 0.0856 & 0.0876 & 0.378 & 0.374 & 0.375 & 0.379 \\
\hline 3.0 & 0.0859 & 0.0852 & 0.0857 & 0.0878 & 0.379 & 0.374 & 0.375 & 0.379 \\
\hline 4.0 & 0.0863 & 0.0853 & 0.0859 & 0.0879 & 0.380 & 0.375 & 0.376 & 0.380 \\
\hline $\begin{array}{c}\text { BEAM } \\
\text { THEORY }\end{array}$ & \multicolumn{3}{|c|}{0.0884} & & \multicolumn{4}{c|}{0.388} \\
\hline
\end{tabular}


varying span $S$ and taper ratio $C_{T} / C_{R}=0.5$ for an uniform distributed load $P$ per unit area and for a concentrated tip load of $P$ units. Non-dimensionalised tip deflections are computed from the results of cases run with: $E=1.0$, $t=1.0, \nu=0.3$ and $C=C_{R}=1.0, C_{T}=0.5$. Mesh idealisations considered are:

(i) entire region modelled by the high precision triangular plate bending elements [1]

(ii) mixed idealisation with plate, transition and beam elements (3,5 and 7 elements are used)

Region $I$ and $I I$ are divided using $S_{\mathrm{p}}=0.05$ and $S_{T}=0.15$. Comparisons with engineering beam theory results indicate convergence of results with increasing aspect ratio and also rapid convergence of results with increasing number of beam elements. It is also interesting to observe that in the mixed idealisation, the monotonic convergence for deflections is from above as the operations involved in the condensation procedures for the transition element do not preserve the convergence from below (a feature of standard displacement models).

Table 2 shows the total bending moment $M_{x}$ resisted by the root section of a straight tapered cantilever plate of varying aspect ratio $L$, taper ratio $C_{T} / C_{R}=0.5, S_{\mathbf{P}}=0.05$, $S_{T}=0.15, E=1.0, t=1.0, \nu=1.0$ under a uniform distributed load $p=1.0$ normal to the plate middle surface. This is obtained by a numerical integration of the bending stresses $M_{x x}$ obtained by the finite element method at the nine nodes at the root (see Fig. 3) using Simpson's quadrature rule. A rapid convergence is seen with increase in number of beam elements, again monotonically, and the results are within acceptable engineering accuracy of the exact value. Also, it is seen that the type of convergence, i.e. from above or below depends on the aspect ratio of the plate.

Figure 4 shows the variation of root bending stress $M_{x x}$, over one half of the straight tapered plate considered above, as a function of the transition element size. We see that when the transition element size is increased (by increasing $S_{T}$ ), thus effectively increasing the region modelled as plate/quasi-plate, the stress at the corner, node 1 of Fig. 3, drops rapdily to the zero-value expected for the boundary layer when the junction angle is acute. However, Table 3 indicates that convergence for the total bending moment at the root is in the reverse direction i.e. with decreasing $S_{T}$, a monotonic convergence of the bending moment is found. Thus in further applications, especially to swept plates, as far as geometry permits, the regions are idealised using $S_{p}=0.05, S_{\mathrm{T}}=0.15$ and only when the

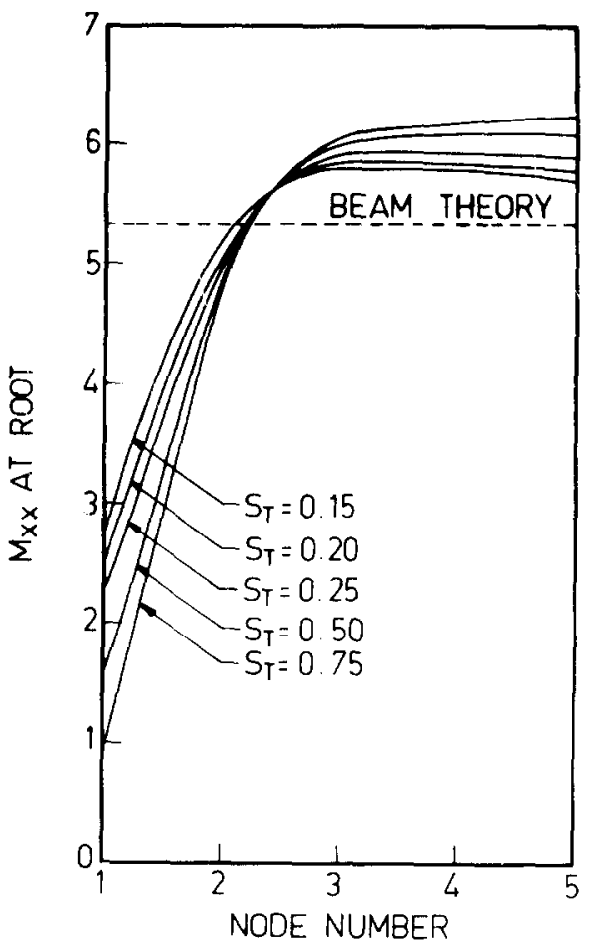

Fig. 4. Root-bending stress variation as function of transition element size for straight tapered plate, $S=4.0, C_{T} / C_{R}=0.5$, $S_{p}=0.05$, under u.d.l, 7 elements in beam region.

Table 3. Total bending moment resisted by root as function of transition element size for straight tapered plate, $L=4.0, C_{T} / C_{R}=$ $0.5, S_{p}=0.05$, under u.d.l., 7 elements in beam region.

\begin{tabular}{|c|c|}
\hline$S_{T}$ & $M_{X}$ \\
\hline 0.15 & 5.317 \\
\hline 0.20 & 5.316 \\
\hline 0.25 & 5.315 \\
\hline 0.50 & 5.303 \\
\hline 0.75 & 5.283 \\
\hline EXACT & 5.333 \\
\hline
\end{tabular}

Table 2. Total bending moment resisted by root as function of $N$, number of beam elements, for straight tapered plate of varying aspect ratio $L, C_{T} / C_{R}=0.5, S_{p}=0.05, S_{T}=0.15$ under u.d.l

\begin{tabular}{|c|c|c|c|c|}
\hline$N$ & $L=1.5$ & $L=2$ & $L=3$ & $L=4$ \\
\hline 1 & 0.745 & 1.327 & 2.994 & 5.350 \\
\hline 2 & 0.746 & 1.327 & 2.994 & 5.330 \\
\hline 3 & 0.746 & 1.328 & 2.993 & 5.325 \\
\hline 5 & 0.767 & 1.329 & 2.992 & 5.319 \\
\hline 7 & 0.747 & 1.329 & 2.992 & 5.317 \\
\hline EXACT & 0.750 & 1.333 & 3.000 & 5.333 \\
\hline ALL \\
COWPER EIF & 0.751 & 1.333 & 2.985 & 5.281 \\
\hline
\end{tabular}


geometry requires it (e.g. at large sweep angles), $S_{T}$ is raised to 0.25 or 0.35 .

As no accurate closed from results are available in the literature, a comparison for a typical example of a swept cantilever plate has been made with an idealisation that used the high precision triangular elements throughout. Figure 5 shows the variation of the bending stress $M_{x x}$ at the root for a swept beam of length $L=4.0, C_{R}=1.0$, $C_{T}=0.5$, angle of sweep $=45^{\circ}, S_{\mathrm{P}}=0.05$ and $S_{\mathrm{T}}=0.15$ for a 7 and 2 beam element idealisation of the beam region. Very good accuracy is observed for the mixed idealisation, even with only 2 bcam clements in region III. In Fig. 6, the variation of transverse deflection and slope of the centre line are compared for the three idealisations and show very good accuracy.

Figure 7 presents the variation of the bending stress over the first four nodes (where the rapid change of bending stress takes place) as the sweep angle $\theta$ is increased for the configuration shown. These stresses are non-dimensionalised as $C M_{x x} / M_{x}$, where $M_{x}$ is the total moment arm

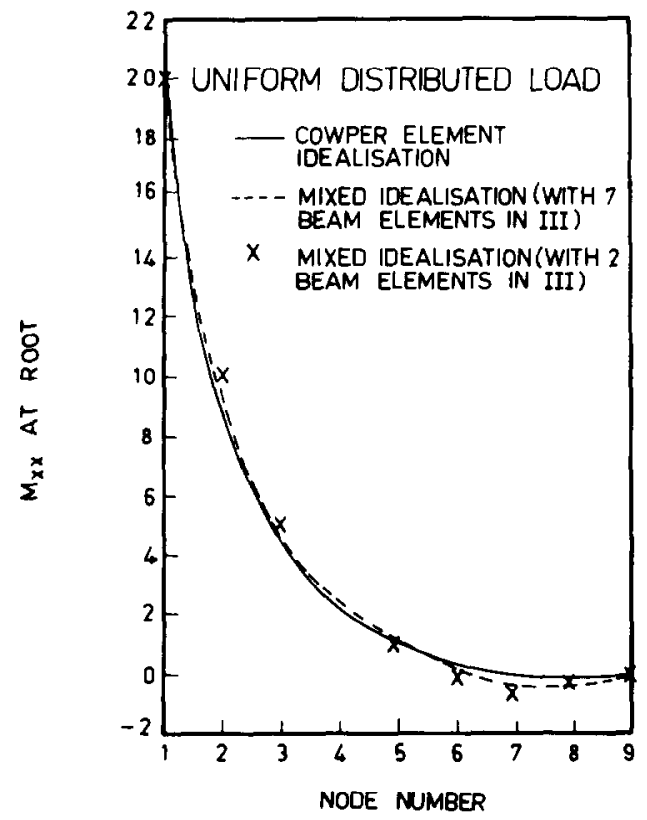

Fig. 5. Variation of root-bending stress for swept beam, $S=4.0$, $C_{T} / C_{R}=0.5$, sweep angle $\theta=45^{\circ}, S_{\mathrm{p}}=0.05, S_{T}=0.15$.
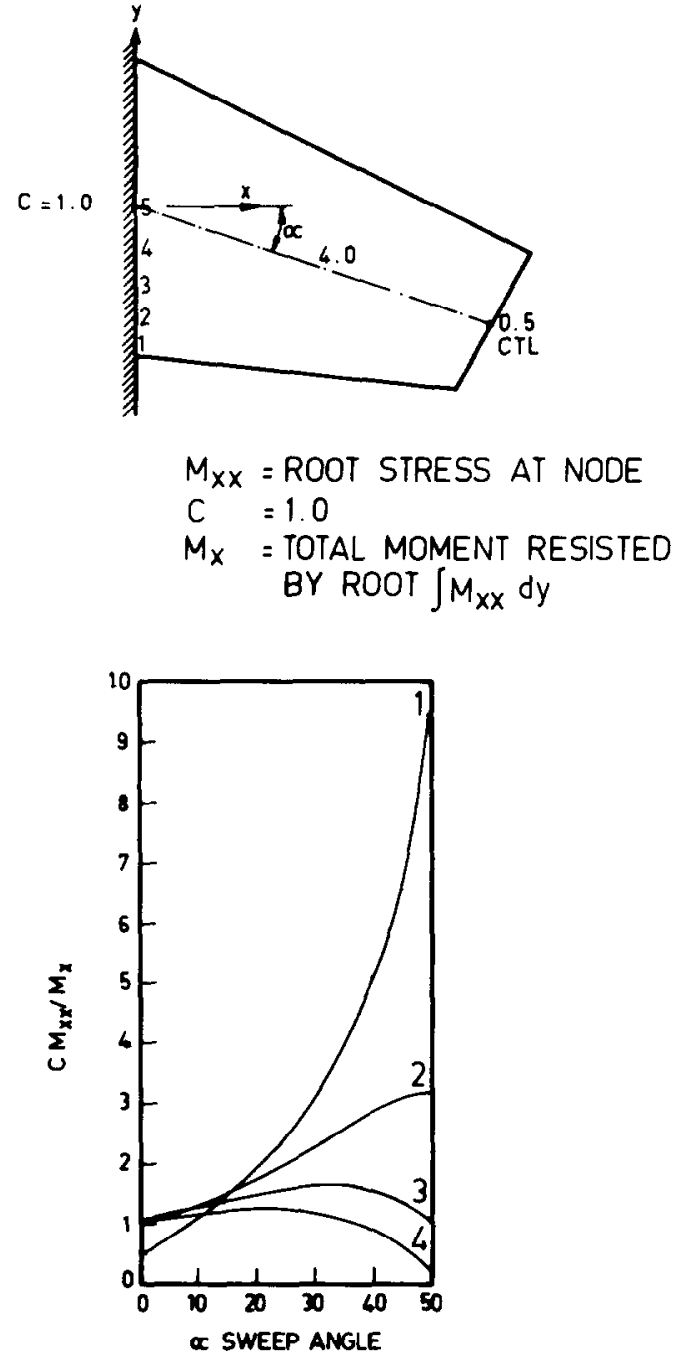

Fig. 7. Root bending stress variation with increasing sweep angle.

resisted at the root due to the action of the concentrated tip load. With increasing sweep, the critical bending stress at the re-entrant corner is seen to increase rapidly.

Figure 8 shows a comparison of the present results for the deflection under a concentrated tip load for the

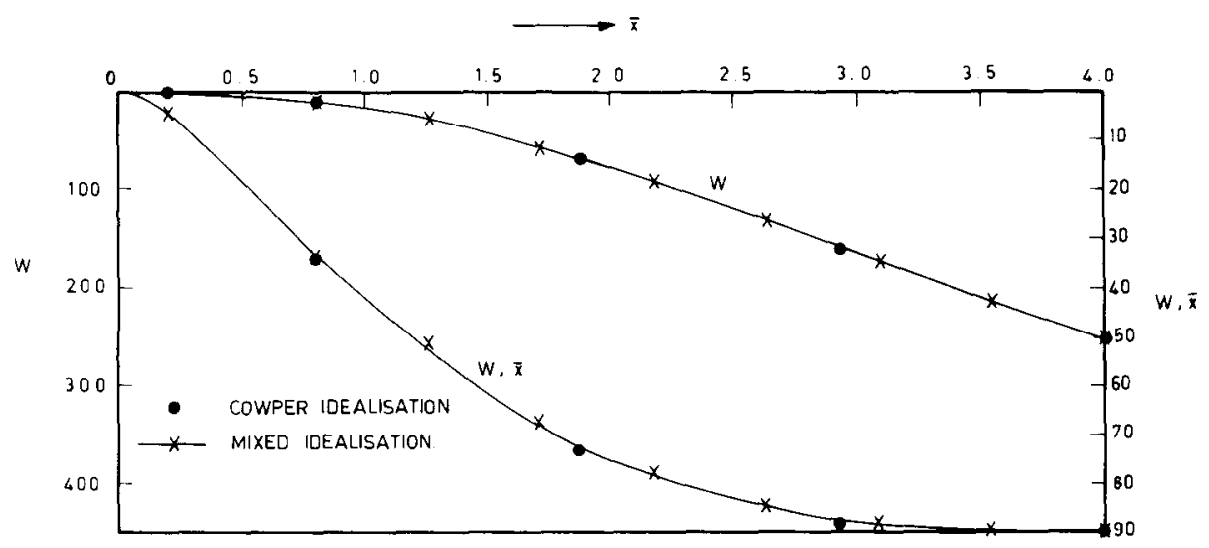

Fig. 6. Variation of deflection and slope along span for swept beam, $S=4.0, C_{T} / C_{R}=0.5$, sweep angle $\theta=45^{\circ}$, $S_{p}=0.05, S_{T}=0.15$. 


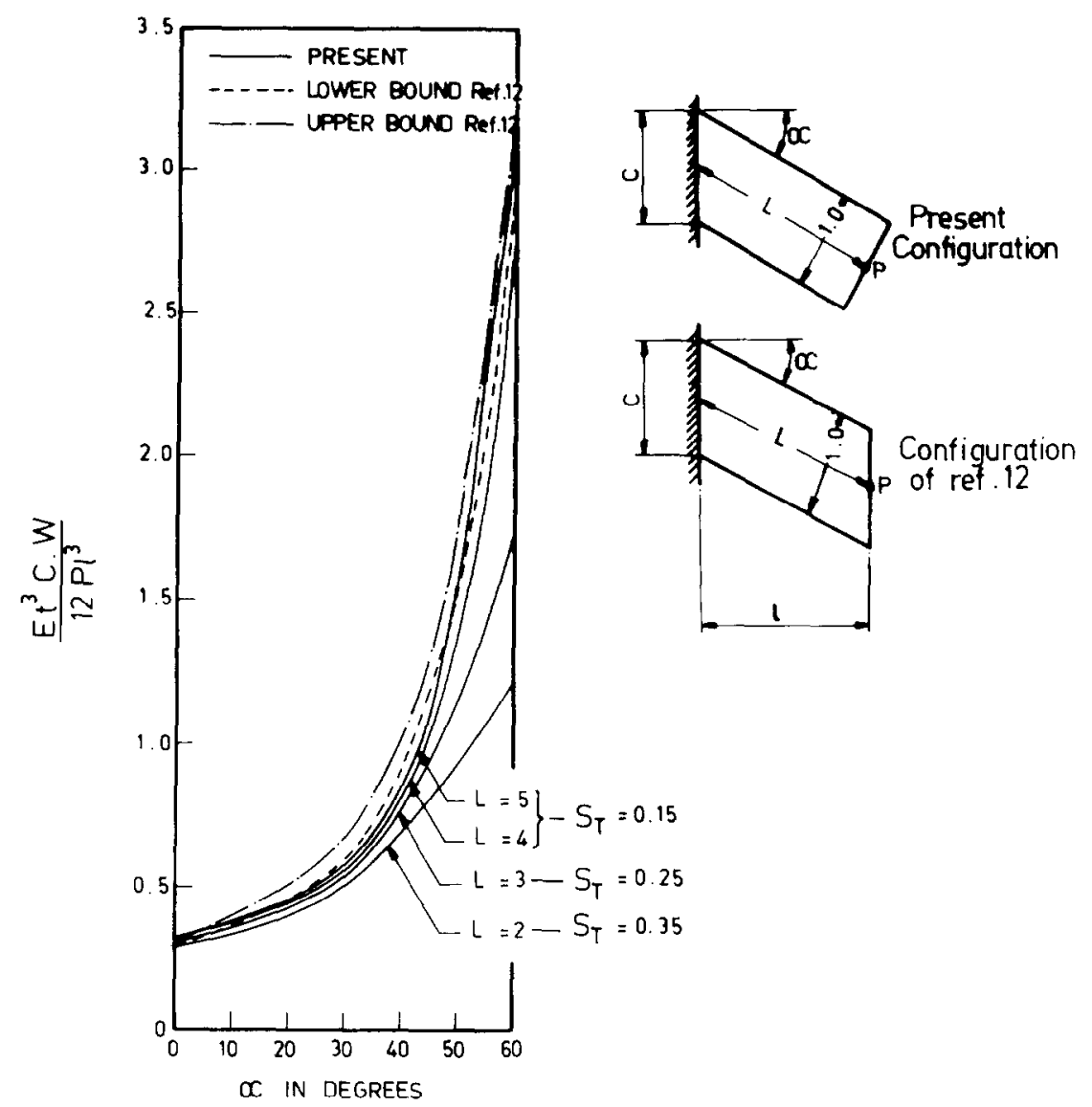

Fig. 8. Non-dimensionalised tip deflection for swept plate under concentrated tip load with increasing sweep angle.

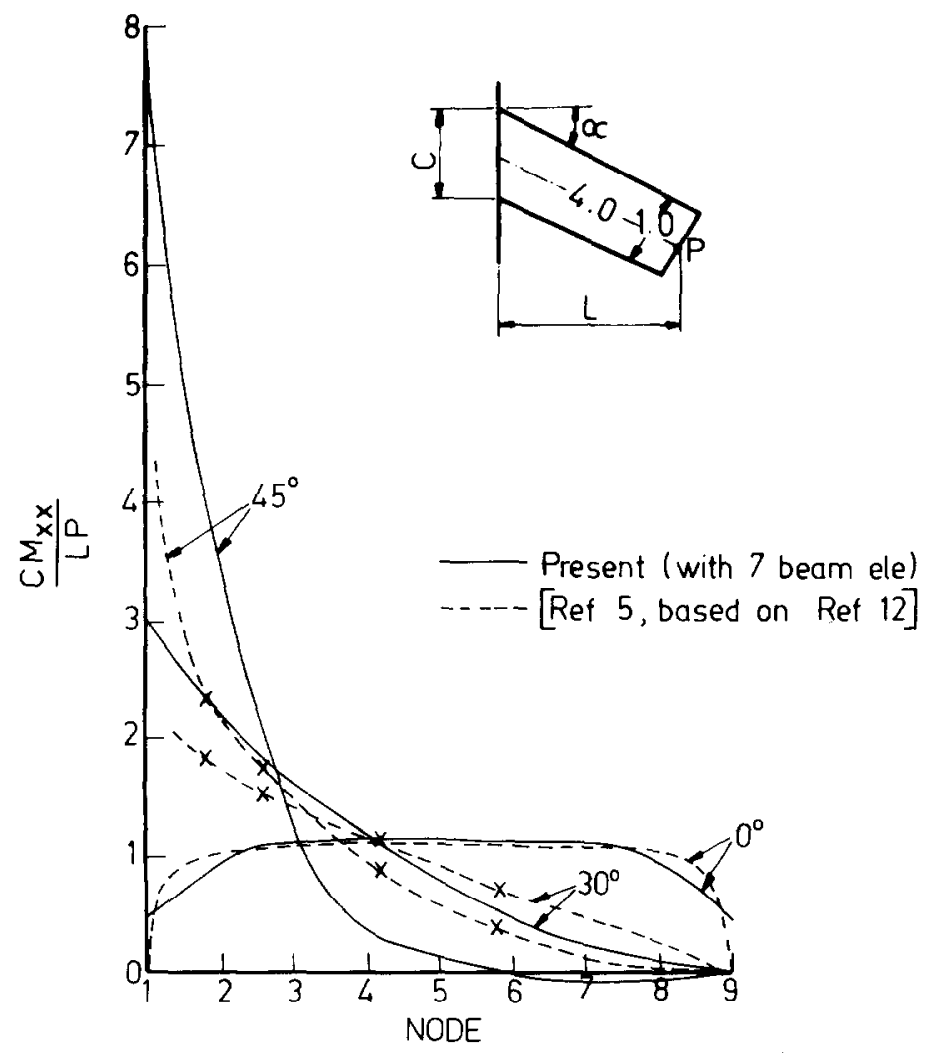

Fig. 9. Root stress variation with sweep angle for constant chord and thickness under concentrated tip load. 
configuration shown with the closed-form formula given for a rhombic swept plate of constant thickness given in Ref. [12]. The two configurations differ somewhat at the tip, in that while the present formulation requires a modelling by beam elements whose edges are normal to the swept centre line and would dictate a tip that is normal to this line, the formulation of Ref. [12] requires an edge parallel to the free stream. However, in all other respects, e.g. distance of the load point from fixed edge and in chord length and span, the two configurations are identical. Thus, invoking St. Venant's principle, it is clear that there should be very little effect on the stress diffusion at regions far away from the load point.

In Fig. 8, the dotted line represents the results as obtained directly from the explicit formula in Ref. [2] and as this theory is based on the assumption of linear deformations in the chordwise direction, this should be good for unswept wings in torsion. In pure beam bending, which would be the nature at low sweep and high aspect ratio, these theoretical results would be off by a factor of $1-\nu^{2}$ and this upper bound is represented by the dasheddotted line. The present mixed finite element idealisation results (solid lines) were obtained with $S_{\mathrm{P}}=0.15$ and $S_{T}=0.15,0.25$ or 0.35 as dictated by the planform and with seven beam elements in the beam region. The results indicate that at low aspect ratio and low sweep, c.g. $S=2,3$, the finite element results are asymptotically close to the theoretical lower bound curve. For higher aspect ratios, $S=4,5$ the results are close to the upper bound curve and the accuracy of the theoretical prediction is good even at large sweep angles. Also, the poorer agreement at low aspect ratio and high sweep could be due to the slight difference in planforms considered in the two models and the non-applicability of the St. Venant's principle. A comparison of the root-stress variation with sweep-angle in Fig. 9 does indicate that although the trends are identical, the finite element method predicts a large but finite stress at the re-entrant corner whereas the analytical solution indicates that indeterminately large stresses are reached in this region.

\section{CONCLUSIONS}

Thus, the principle of using selectively chosen elements over different regions of the plate can allow the combination of high-precision elements where required, with simpler elements, together with transition elements to match the two disparate element types in inter-phase regions shows that a considerable reduction in problem size and solution time can be achieved. The practicality of obtaining solutions based on this concept has been demonstrated by its application to swept cantilever plates. It is quite simple to envisage an extension of this problem to the use of a combination of $3 \mathrm{D}$ brick elements at the root for accurate root stress prediction through the root-thickness, and plate and beam elements with the appropriate transition elements at regions far away from the root. A similar extension is possible with shell structures also. Thus accurate stress predictions at the root, without the enormous increase in problem size, that can be expected if the entire structure is modelled by $3 \mathrm{D}$ brick elements, is possible.

Acknowledgements--The authors wish to acknowledge with pleasure the help and encouragement given for this work by Prof. A. V. Krishna Murthy, Indian Institute of Science, Ban- galore, India. The authors would also like to record their appreciation to Dr. G. Prathap, Scientist, National Aeronautical Laboratory, for the valuable assistance rendered during the course of this work.

\section{REFERENCES}

1. G. R. Cowper, E. Kosko, G. M. Lindberg and M. D. Olson, Static and dynamic application of a high precision triangular plate bending element. AIAA J. 7, 1957-1965 (1969).

2. B. R. Somashekar, A simple selectively integrated torsionflexure coupled tapered beam element with transverse shear deformation. Computers \& Structures (to appear).

3. B. R. Somashekar and G. Prathap, On boundary condition's suppression in high precision triangular plate elements. Proc. 26th Congress of ISTAM, Coimbatore (Dec. 1979).

4. M. L. Williams, Surface stress singularities resulting from various boundary conditions in angular corners of plates under bending. U.S. Nat. Congr. Appl. Mech., 325 (1951).

5. M. L. Williams, A review of certain analysis methods for swept wing structures. J. Aeronautical Sci. 19, 615-629 (1952).

6. A. L. Lang and R. L. Bisplinghoff, Some results of sweptback wing structural studies. J. Aeronautical Sci. 18, 705 (1951).

7. P. E. Bisch and J. B. Mattson, The slice mehod of box-beam analysis as applied to a model swept back wing. North American Aviation Rep. No. NA-47, 136 (Feb. 1974).

8. D. B. Hall, Method of stress analysis of a swept back wing. Glenn L. Martin Rep. No. SDR-37 (Oct. 1947).

9. E. Sechler, M. L. Williams and Y. C. Fung, An initial approach to the overall structural problems of swept wings under static loads. J. Aeronautical Sci. 17(11), 639 (1950).

10. Y. C. Fung, Stress and deflection analysis of swept plates. Air Force Techn. Rep. No. 5761, Part 2 (June 1949).

11. H. U. Schucreh, Theorctical analysis of twist and deflection of swept structures with application to a solid delta wing of double wedge cross-section subjected to a uniform load. Structures Memorandum No. 6 AESRL MIT (April 1951).

12. E. Reissner and M. Stein, Torsion and transverse bending of cantilever plates. NACA Tech. Note 2369 (1951).

13. H. J. Plass, J. H. Gaines and C. D. Newson, Application of Reissner's variational principle to cantilever plate deflection and vibration problems. J. appl. mech. 29, 127-135 (1961).

14. A. Coull, The direct stress analysis of swept cantilever plates of low aspect ratio. Aircraft Engng 37, 182 (1965).

15. D. J. Dawe, Parallelogramic elements in the solution of Rhombic cantilever plate problems. J. Strain Analyses 1, 223-230 (1966).

\section{APPENDIX}

Derivation of $(T)$ matrix

In Fig. 10, $A B$ is the line of transition from the transition element element 0 the adjacent beam element. We must relate the 6 global d.o.f. ( $W W, x, W_{, y}, W_{, x y} W_{, y y}$ ) at each of the nodes $1-5$ to the 5 d.o.f. offered by the beam element at node 3 . We consider first, the global degrees of freedom and expand it as a Taylor series expansion about node 3 such that

$$
W_{(x, y)}=W_{3}+x W_{3, x}+y W_{3, y}+\frac{x^{2}}{2} W_{3, x x}+x y W_{3, x y} .
$$

By evaluating the r.h.s. and its first and second derivatives at all

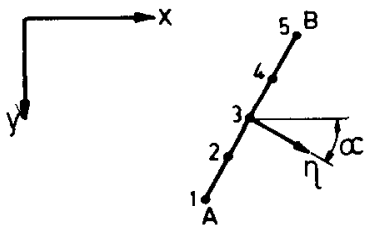

Fig. 10. Geometry of right edge of transition element. 
5 nodes, one can set up the 305 transformation matrix [T]. A typical submatrix of [T] at node $i$ on line $A B$ would be

$$
\left[T_{i}\right]=\left[\begin{array}{ccccc}
1 & x_{i} & y_{i} & \frac{x_{i}^{2}}{2} & x_{i} y_{i} \\
0 & 1 & 0 & x_{i} & y_{i} \\
0 & 0 & 1 & 0 & x_{i} \\
0 & 0 & 0 & 1 & 0 \\
0 & 0 & 0 & 0 & 1 \\
0 & 0 & 0 & 0 & 0
\end{array}\right]
$$

